\title{
La enseñanza de una metodología integral de colaboración para la gestión de proyectos en construcción civil: la metodología BIM en la educación superior
} \author{
its Diaspora

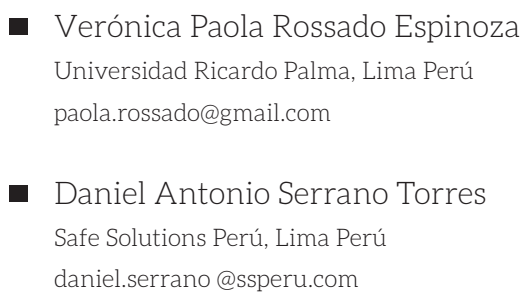

Scientific Approach to the Project in a Computational Perspective of Architecture: The Hochschule für Gestaltung-Ulm and

\begin{abstract}
BIM methodology is a process that make possible to integrate the construction project on a $3 D$ model. It contains the necessary information, optimizing stages, allowing early analysis and product virtualization. This methodology, reduces the time of implementation, anticipates the decisions making and improves productivity. The project requires the coordination of various professionals such as architects, civil, electrical, structural engineers, contractors and administrators, who needs specialized teaching by experts in BIM methodology. There are few professionals who are truly qualified to lead or carry out an entirely project whit this methodology.
\end{abstract}

Keywords: Arquitectura, Construcción, Modelado BIM, Enseñanza BIM, Proyectos Digitales, Proyectos Cooperativos

\section{Introducción}

Todo proceso de desarrollo de un proyecto de construcción civil, involucra el trabajo de un equipo de profesionales y especialistas, que intervienen en determinadas etapas del proyecto, donde su organización y eficiencia garantizará el éxito y la calidad de la obra.

Dentro de este proceso multidisciplinario, arquitectos, ingenieros civiles, eléctricos, estructurales y contratistas, deben coordinar e intercambiar ideas para el óptimo desarrollo del proyecto. Cada especialista es responsable de un determinado aspecto, buscando la solución más adecuada en el menor tiempo posible. "Es necesario un trabajo en conjunto y un espacio de colaboración, donde cada área pueda contribuir con los datos necesarios para lograr un objetivo en común". (Eastman, 2008).

Es innegable la importancia del diálogo y el trabajo colaborativo del equipo de expertos para la efectividad de un proyecto, aunque en ocasiones resulta ser una tarea difícil. Será preciso contar con un espacio o plataforma de colaboración, donde los agentes involucrados compartan sus conocimientos en un proceso integral y eficiente.

Esta plataforma debe permitir el trabajo en equipo mediante la aplicación de una metodología adecuada, para intervenir y trabajar sobre un mismo modelo que contenga datos, anticipando problemas y resolviendo interferencias que puedan ocurrir en el proceso. Este modelado de la información, es conocido como metodología BIM (Building Information Modeling), mediante el cual, en el modelo $3 \mathrm{D}$, se integra la información requerida por todas las especialidades, para el desarrollo eficiente y organizado de un proyecto de construcción.

Actualmente, las etapas de diseño de un proyecto se ejecutan de manera consecutiva y lineal (una después de la otra) lo que puede ocasionar conflictos, aumento de costo y tiempo. Son muchos los profesionales que intervienen y deben coordinar en el desarrollo, debiendo analizar numerosos factores para alcanzar un resultado eficiente, tanto durante el diseño, la gestión, construcción o mantenimiento de la obra.

En Europa y Norteamérica, son varios los casos de éxito de proyectos ejecutados con BIM, pero requieren de un mayor número de profesionales capacitados para atender sus necesidades. En Perú, grandes empresas constructoras están iniciando sus proyectos con esta plataforma, sin embargo, son pocos los profesionales con conocimientos y experiencia para dirigir o realizar un proyecto íntegramente con BIM.

\section{Ejecución de un Proyecto BIM}

Un proyecto de construcción ejecutado en BIM, contempla varias etapas, éstas se denominan Fases del Ciclo de Vida del Proyecto (PLPs, del inglés Project Lifecycle Phases) e 
incluyen actividades tanto de pre-construcción (planificación, estimación de costos), como de post-construcción (ocupación y mantenimiento de la instalación) (Razwi, 2008).

Durante este ciclo, se consideran tres fases principales: diseño, construcción y operaciones, que involucran distintos especialistas. Cada uno dotará al modelo 3D de toda la información necesaria para su realización, esto reduce el tiempo, optimiza recursos, anticipa la toma de decisiones y mejora la productividad. Según Succar, para que el proyecto BIM se desarrolle de forma ordenada, se debe ejecutar en tres etapas: modelado paramétrico de objetos, colaboración basada en el modelo, e integración en la red. (Succar, 2009).

\section{Modelado basado en objetos}

El modelado se obtiene mediante un software de diseño paramétrico basado en objetos 3D, generando modelos uni-disciplinarios. Los datos permiten la exportación de la documentación para su interpretación, organización, gestión y administración de actividades en un diagrama de flujo.

\section{Colaboración basada en el modelo}

En esta etapa, cada disciplina aporta datos que enriquecen el proyecto, tanto para la construcción como para la gestión de la obra en forma colaborativa. La información del diseño arquitectónico, aportará al diseño estructural y a las especialidades. Las etapas se van superponiendo y realizando en paralelo con las otras, gestionando el tiempo y las actividades en cada momento del proyecto.

\section{Integración en la red}

El modelo paramétrico y la información que contiene se evalúa y analiza en la red bajo un proceso lineal, generando un flujo en base a las actividades y la coordinación entre los agentes ejecutores. Ésta deberá realizarse en forma multidisciplinaria e integral, así por ejemplo, en la etapa de diseño arquitectónico, intervendrán factores de diseño estructural, instalaciones y de construcción, donde se optimicen los recursos profesionales y materiales.

\section{La Enseñanza del BIM}

La enseñanza de la metodología BIM, se viene realizandoa nivel mundial desde hace poco tiempo. Algunas universidades europeas promueven programas de posgrado que incluyen la gestión y administración de proyectos con metodología BIM (Master BIM). El mercado norteamericano ofrece también una amplia oferta de cursos, formando y certificando expertos en proyectos BIM. Sin embargo, en Latinoamérica su enseñanza está aún en inicio.

Estos cursos y programas de máster están orientados a todo profesional de la construcción: arquitectos, ingenieros, promotores, gerentes de proyectos y contratistas, que deseen organizar su equipo de trabajo bajo una sola plataforma de cooperación e integración. Mediante la gestión BIM en la empresa, se busca incrementar las ganancias y la eficiencia de la construcción, optimizando los costos del proyecto.

\section{Programa de Especialización}

La necesidad de profesionales en el uso del BIM en Perú, sumado a los casos de éxito a nivel mundial, incentiva la propuesta de creación de un programa de especialización a nivel superior. Este programa deberá estar orientado al mercado peruano, con contenidos curriculares que contemplen una solución y visión a nivel latinoamericano.

Este programa estará dirigido a los egresados y profesionales de la arquitectura, ingeniería y toda persona relacionada con la construcción, de tal manera que los egresados adquieran el conocimiento de la herramienta BIM como una metodología que les permita mejorar la comunicación y coordinación de un proyecto de construcción en todas sus etapas.

El perfil del programa estará centrado principalmente en la realidad nacional y las actividades se enfocarán en buscar soluciones a la problemática y las necesidades del mercado peruano. Se pondrá un interés especial a los avances y propuestas realizadas a nivel mundial, con el fin de encontrar sus repercusiones y mejoras en el medio latinoamericano.

\section{Procedimientos Metodológicos}

Para dar inicio a la investigación, se realizó un estudio de las principales universidades del país, especializadas en la enseñanza de carreras profesionales relacionadas a la construcción civil, a fin de conocer su forma de enseñanza y orientación de la metodología BIM.

Se estudiaron los planes curriculares de las principales universidades del país, especializadas en la enseñanza de ingeniería civil y arquitectura. A partir de la revisión de sus contenidos, se evidencia que éstos se orientan al dibujo CAD, la arquitectura digital con Revit Architectural, no se llega con profundidad a la enseñanza del trabajo cooperativo. A nivel de pre grado, los estudiantes aprenden el manejo de herramientas digitales de la especialidad, de acuerdo a lo requerido por su perfil profesional.

Como parte del estudio de factibilidad para dar inicio al Programa de Especialización, se tomó una muestra de 150 egresados de arquitectura en los últimos 5 años, a quienes se aplicaron encuestas y entrevistas, demostrando que la mayoría no tiene conocimientos de la metodología BIM, sin embargo, se muestran interesados en su aprendizaje. Del mismo modo, a fin de difundir esta nueva forma de trabajo, se vienen realizando seminarios de capacitación y charlas informativas, donde se tiene la oportunidad de dar a conocer sus beneficios y ventajas.

A nivel empresarial, se entrevistaron a los jefes de proyecto, promotores y responsables de obra de las principales empresas constructoras del país, quienes manifestaron la necesidad de que su personal domine la herramienta. Conscientes de las ventajas del BIM y la necesidad inmediata de su aplicación, algunos optan por 
la capacitación en el exterior, otros contratan instructores particulares para su aprendizaje personalizado. Sin embargo, se hace necesaria una capacitación orientada al mercado nacional, donde se discutan y solucionen los problemas con otros especialistas peruanos.

El Comité BIM del Perú, con el fin de unir esfuerzos en la promoción y divulgación de las ventajas de aplicar esta metodología, realiza un congreso anual donde invita a especialistas internacionales $\mathrm{y}$ nacionales a debatir $\mathrm{y}$ compartir sus experiencias. Este evento ha tenido mucha aceptación entre las empresas y profesionales, sin embargo, se busca obtener un mayor número de participantes y auspiciadores en cada nuevo congreso.

\section{Implementación del Programa de Especialización}

El programa es pionero en su tipo en Perú, estará dirigido a los egresados de arquitectura e ingeniería en sus distintas ramas y a toda persona relacionada al sector construcción. Tiene por objetivo preparar al profesional en el manejo eficiente de la obra de construcción, mediante la metodología BIM, haciendo posible la comunicación fluida y el trabajo colaborativo interdisciplinario. Su eficacia deberá estar evidenciada en el ahorro de tiempo, la optimización de la gestión en la construcción y la rentabilidad del proyecto.

Los contenidos y plan curricular del "Programa de Especialización en Gestión de Proyectos con Metodología BIM", se basan en la realidad y situación actual de la construcción y las posibilidades del mercado peruano, considerando que aún no se establecen políticas o normativas para la construcción estandarizada.

Se analizaron ejemplos de casos exitosos de programas de Maestrías y Diplomados realizados en Universidades europeas, sin embargo, las necesidades del mercado latinoamericano son diferentes, por lo que se reestructuró el programa en base a la realidad nacional.

La propuesta final de contenido curricular del Programa, se sometió a consulta y discusión por parte de profesionales con experiencia en la ejecución de proyectos BIM en el Perú, quienes, junto con docentes universitarios y expositores especialistas de las diferentes ramas, fueron invitados a formar parte de la cátedra del programa, garantizando una enseñanza de calidad.

\section{Resultados}

El programa toma especial importancia en la aplicación práctica de la metodología, donde se requiere que el alumno experimente el trabajo colaborativo y multidisciplinario. La enseñanza de diseño en la Facultad de Arquitectura y Urbanismo de la Universidad Ricardo Palma, se distingue por emplear un método de enseñanza estructurada en base a talleres, donde se forman grupos de trabajo que incluyen alumnos de varios niveles o ciclos. La metodología de talleres se utilizará para organizar los ejercicios prácticos en el

\section{Programa de Especialización BIM.}

\section{Metodología de enseñanza BIM en la FAU}

Dado que la mayoría de los participantes son profesionales con experiencia en arquitectura, ingeniería y/o construcción, se hace posible la formación en grupos multidisciplinarios, organizados en base a talleres, enriqueciendo el desarrollo del Programa. Se contará con dos profesores por sesión de taller: un arquitecto y un ingeniero de la especialidad, de acuerdo al tema a tratar.

En cada sesión, se aplicarán las siguientes estrategias de enseñanza-aprendizaje:

1. Las clases combinarán sesiones de teoría con talleres prácticos, una hora de teoría y una hora de práctica por clase. 2. Se mantendrá un sistema continuo de evaluación por medio de un docente expositor y el apoyo de un jefe de prácticas, en grupos de no más de 20 alumnos por aula.

3. El docente motivará el desarrollo de debates y el intercambio de información entre los grupos, buscando el mejoramiento continuo y la autoevaluación.

4. Se aplicarán métodos de enseñanza a partir del estudio de casos y el aprendizaje en base a problemas.

5. Se asegurará la comunicación continua entre profesores y alumnos, con el apoyo del aula virtual (Moodle) y el trabajo en la nube.

6. Se realizarán actividades de refuerzo a través de material didáctico, guías de clase, videos, separatas, manuales y fuentes confiables de investigación web.

7. Se organizarán prácticas individuales y grupales, a fin de garantizar el cumplimiento de tareas y la asimilación de conceptos.

8. Los alumnos contarán con el apoyo constante de los expositores, por medio de asesorías virtuales; se verificará la correcta organización del equipo de taller y el avance de trabajos.

9. Se fomentará en los participantes la investigación y la ejecución de nuevos proyectos más complejos, buscando el mejoramiento continuo.

\section{Estructura de contenidos}

El Programa de Especialización se estructuró en base a 5 unidades de aprendizaje:

1. Coordinación virtual: donde el alumno tendrá un amplio conocimiento sobre la filosofía BIM, sus componentes y plataformas empleadas en el mercado peruano. Conocerá la historia del BIM y los casos de éxito a nivel mundial. Aprenderá a realizar de manera correcta la coordinación entre los profesionales involucrados en el proyecto, desde el diseño hasta la ejecución de la obra. Conocerá como realizar la planificación y gestión de vida del proyecto dentro de la plataforma BIM.

2. Planificación: el alumno podrá planificar y gestionar un proyecto de manera colaborativa, aplicando un trabajo profesional multidisciplinario (arquitectura, estructuras, instalaciones, construcción y gestión). Realizará 
adecuadamente el flujo de actividades entre las diferentes especialidades durante las etapas del proyecto.

3. Pre diseño: el alumno realizará el modelado paramétrico para arquitectura y las especialidades. Realizará la interacción del diseño arquitectónico con el diseño estructural, de instalaciones (MEP) constructivo y de gestión.

4. Desarrollo y documentación: el alumno realizará de manera eficiente la documentación, logística y elaboración de planos a nivel de obra. Podrá realizar el análisis económico, costos y presupuestos.

5. Construcción y operación: el alumno aprenderá a analizar y resolver interferencias, para un adecuado control de la obra, operación y seguridad. Será capaz de realizar simulaciones y virtualizaciones de manera que garantice la efectividad de la construcción y optimice los tiempos de ejecución.

El Programa de Especialización tendrá una duración de 180 horas académicas. Al finalizar el Programa de Especialización, el alumno será capaz de desempeñarse como Coordinador BIM, de manera que pueda planificar, gestionar y ejecutar de manera eficiente un proyecto de construcción civil, optimizando las actividades de diseño, gestión y construcción, mejorando su rentabilidad.

\section{Discusión}

A pesar de los esfuerzos que realizan los comités científicos, la metodología BIM no se aplica en todo su potencial en país. El trabajo aportará un material importante para la difusión e implementación de la enseñanza de la metodología y la herramienta BIM a nivel superior. Se busca capacitar a profesionales y egresados de universidades e institutos superiores, ante el aumento de la demanda de importantes empresas que desean contar con profesionales BIM.

El sistema BIM como una metodología y herramienta de gestión, comunicación y trabajo colaborativo, está iniciándose, los egresados del programa de especialización tendrán el reto de enfrentar el difícil mercado proyectual tradicional. Será necesario formar profesionales con visión de futuro, que demuestren que la aplicación de esta metodología es necesaria para sus empresas y para el país.

"Estamos en el inicio de una etapa que transformará la industria de la construcción y las instalaciones, que cambiará profundamente la manera en que la humanidad interactúa con su entorno construido. Este cambio, producto del modelado de la información, permitirá a las personas una experiencia de simulación virtual antes que su manifestación física." (Underwood, 2010).

La metodología BIM “...está revolucionandola industria de la construcción. Reemplazando las herramientas de dibujo 2D. El BIM es el proceso colaborativo usado por la gran industria de la construcción, incluyendo la comunidad de AEC/FM (Architecture/ Engineering/ Construction and Facilities Management) (Arquitectos, Ingenieros, constructores y Administradores de la Construcción), propietarios y stakeholders" (Epstein, 2012)

Patrick MacLeamy, (Chief Executive Officer of HOK) afirma: "BIM es la primera tecnología verdaderamente global la construcción digital y pronto será desplegado en todos los países del mundo. Es un "cambio de juego" y tenemos que reconocer que está aquí para quedarse - pero como toda innovación, representa tanto un riesgo, como una oportunidad". (MacLeamy, 2014). "A nivel internacional, la metodología BIM está siendo más difundida y aplicada, pronto causará un mayor impacto en el país, y aumentará la demanda de capacitación apropiada en el tema". (HOK, 2014). HOK es una firma de diseño con una red de 24 oficinas en todo el mundo que ofrece soluciones creativas, producto de un proceso de colaboración multidisciplinaria.

La realización del programa permitirá, por medio de sus egresados, difundir entre las empresas constructoras y estudios de arquitectura del país, las ventajas de esta metodología, contribuyendo en el desarrollo y mejoramiento de la construcción a nivel nacional.

\section{Agradecimientos}

Se agradece a la Facultad de Arquitectura y Urbanismo de la Universidad Ricardo Palma, a la Empresa Safe Solutions y a todos los profesionales expertos que colaboraron en la realización de la presente propuesta de capacitación.

\section{Referencias}

BIM ThinkSpace (2008) Effects of BIM on Project Lifecycle Phases. Obtenido de: http://www.bimthinkspace. com/2008/11/effects-of-bim-on-project-lifecycle-phases. html

Epstein, E. (2012). Implementing Successful Building Information Modeling. Artech House.

Eastman, Ch.; Teicholz, P.; Sacks, R.; Liston, K. (2008). BIM Handbook: A Guide to Building Information Modeling for Owners, Managers, Designers, Engineers and Contractors. John Wiley \& Sons.

Escuela de posgrado Universidad Europea. Posgrado de experto en gestión de proyectos, metodología BIM. Obtenido de: http://universidadeuropea.es/estudios-universitarios/ postgrado-de-experto-en-gestion-de-proyectos-metodologiabim

HOK. Design Annual 2014. ORO Editions. Publishers of Architecture, Art and Design. (2014). Obtenido de: http:// www.hok.com/uploads/2014/09/03/2014hok-designannual.pdf

HOK. Tall Buildings. ORO Editions. Publishers of Architecture, Art and Design. Obtenido de: http://www.hok.com/ uploads/2014/05/08/hok-tall-buildingsweb.pdf

Project Production Institute. PPI. Obtenido de: http:// projectproduction.org/

Parsons Brinckerhoff. (VDC) Project and BIM services. Obtenido de: www.pbworld.com/capabilities_projects/ visualization/bim_vdc.aspx 
Razwi, S. Bim and the Process Improvement Movement. Building a case for a combined BIM-CMMI Framework. BIM ThinkSpace (2008). Obtenido de: http://changeagents. blogs.com/thinkspace/files/BIM_ThinkSpace_The_Process_ Improvement_Movement_by_Sohail_Razvi.pdf.

Stanford University. Center for Integrated Facility Engineering. CIFE. Obtenido de: http://cife.stanford.edu/

Succar. B. (2009). Building Information Modelling Framework: AResearchand delivery Foundation for Industry Stakeholders. Automation in Construction. University of Newcastle, NSW Australia. Obtenido de: https://www.academia.edu/170356/ Building_Information_Modelling_framework_a_research_ and_delivery_foundation_for_industry_stakeholders
Succar. B. The Five Components of BIM Performance Measurement. University of Newcastle, NSW Australia. Obtenido de: https://www.academia.edu/227815/The_Five_ Components_of_BIM_Performance_Measurement

The Future of BIM: Looking beyond 2016. University of Salford Manchester. Obtenido de: http://www.salford.ac.uk/ onecpd/courses/the-future-of-bim-looking-beyond-2016

Underwood, J. Isikdag, U. (2010). Handbook of Research on Building Information Modeling and Construction Informatics: Concepts and Technologies. Information Science Reference. New York.

24 Estudio Lab Centro de formación Autodesk. Obtenido de: http://www.24studiolab.com/\#/ 